\title{
How Egocentric Biases Maintain Social Anxiety: A Literature Review Kyra Mingus*, Psychology
}

\begin{abstract}
Biases and heuristics are mental shortcuts that help guide our daily decision making and cognitive processing but can often lead us astray when they account for inaccurate or misinterpreted information. In this review I aim to understand how the spotlight effect (Gilovich et al., 2000), the overestimation of how attentive others are to our actions, and the illusion of transparency (Gilovich et al., 1998), the overestimation of how easily others can discern our internal state, maintain social anxiety by disrupting the anchoring component these shortcuts rely on. Through a detailed analysis of major research conducted by Brown and Stopa (2007) and Haikal and Hong (2010), I was able to synthesize the empirical findings, discuss clinical implications, and propose future directions for research.
\end{abstract}

As humans we are innately driven by the psychological need to satisfy our ego, the subconscious mediator between impulsivity and morality, but how is the ego influenced when we are put into social situations? The ego, in tandem with many cognitive biases and heuristics, is at work during the decision-making process, but by employing these shortcuts, relative fallacies are often committed, resulting in poor decisions based on inaccurate judgements. Specifically, egocentric biases influence our perception of ourselves and how we expect others to perceive us, which can lead to a distorted and unrealistic sense of self. A strong sense of self is an invaluable tool in navigating our social world because it fosters a positive self-image, high self-esteem, and a more congruent relationship between one's self-image and their ideal self (Rogers, 1974). However, a strong sense of self is often hard to achieve when we commit fallacies associated with egocentric biases. These include the spotlight effect and the illusion of transparency, as they rely on perceived information that is inaccurate. The spotlight effect perpetuates distortion by overestimating how noticeable our actions are when around other people (Gilovich \& Savitsky, 2000). In contrast, the illusion of transparency describes how noticeable we believe our internal state is to others (Gilovich et al., 1998). With both biases distorting our perceptions and increasing our self-awareness, they create a clear formula for anxiety. This is concerning for those at risk or currently diagnosed with social anxiety disorder because those individuals already view

*Kyra Mingus is a fourth-year student at finishing her B.S. in psychology and minors in sociology and legal studies. She is aspiring to be a clinical social worker with specific interests in childhood trauma, family dynamics, interpersonal violence, and psychopathology. In her free time, Kyra enjoys making art and spending time with her friends and family. Please direct correspondence to kmingus2@uoregon.edu. 
themselves from an incongruent sense of self created by their disorder which is viscously perpetuated by the spotlight effect and the illusion of transparency. To understand the clinical implications of this phenomenon, I will describe and synthesize the findings of the two main studies that currently sustain the body of knowledge specifically addressing these biases in the context of social anxiety.

\section{THE SPOTLIGHT EFFECT}

Initially explored by Newell (1957), heuristics are mental shortcuts that simplify the decisionmaking process by reducing mental effort. He highlights the innate limitations faced in rational decision making, such as taking the time and mental effort to weigh the costs and benefits of all alternatives to produce purely rational decisions, a process most people would not use in their daily lives. Tversky and Kahneman (1982) built on Newell's research and introduced the study of heuristics by proposing three types of heuristics in their initial research: availability, representativeness, and anchoring-and-adjustment. Tversky and Kahneman defined the availability heuristic as a cognitive shortcut used when making probability and frequency-based judgments depending on how easily "available" information regarding the decision is recalled (1982). Similarly, the representative heuristic also employs known information, but instead of recalling specific instances, it uses pre-conceived prototypes, or the subjectively "perfect" mental image of something, to gauge probability (Tversky \& Kahneman, 1982). Finally, anchoring-andadjustment is defined by Tversky and Kahneman as estimating frequency, probability, and value in relation to an implicit reference point, or anchor (1982). They found that people adjust away from the anchor to draw their conclusions, but unfortunately, the initial anchor is often inaccurate, causing erroneous adjustments. A common example of the anchoring-and-adjustment process is observable in car sales negotiation, where the "sticker" price for a used car is often higher than the actual market value of the car. Car sales agents use this to their advantage because negotiations are then in relation to the unreasonably high anchor price, fooling the buyer into believing they are getting a good deal if they can negotiate a price under that anchor. In reality, the buyer is most likely still paying above market value and similar scenarios are not uncommon in the real world, and in our perception of ourselves.

Additional research conducted in 2000 by Gilovich et al. built off of Ross and Sicoly's (1979) research on responsibility allocation which illustrated how individuals are so focused on their own contribution in a joint task that when asked "Who did how much?" they were biased towards their contributions. Gilovich et al. (2000) then proposed a similar effect, the spotlight effect, describing how people judge the salience of their own actions by others. They further supported the spotlight effect through Tversky and Kahneman's (1982) anchoring theory, arguing that an inaccurate anchor causing an insufficient adjustment creates it. Ultimately, the spotlight effect is rooted in our own egocentric nature inflating our perception of our actions, which can become a uniquely inaccurate anchor based on how salient we believe those actions are to others.

\section{THE ILLUSION OF TRANSPARENCY}


Similarly, the illusion of transparency is also rooted in Tversky and Kahneman's (1982) anchoring theory. Research conducted in 1998 by Gilovich et al. (prior to their exploration of the spotlight effect) helped expose the mechanics of this concept and its relationship to anchoring. The researchers defined the illusion of transparency as the "tendency to overestimate the extent to which others can read one's internal state" (Gilovich et al., 1998, p. 332). While the spotlight effect is the overestimation of the visibility of our actions in a social setting, the illusion of transparency is the overestimation of how our internal state "leaks" out for outsiders to notice. For example, if you were in a hurry to walk to class and trip on the sidewalk, you may feel embarrassed, which then becomes the anchor situational adjustments are made from. How noticeable to others you believe your fall to have been is distorted by the spotlight effect, but if you believed others knew you were embarrassed by it, this would be the illusion of transparency at work. It is important to distinguish that the spotlight effect disrupts external perceptions, while the illusion of transparency affects internal perceptions.

In addition to defining the illusion of transparency, Gilovich et al. (1998) also found that those who are highly self-conscious, giving them a heightened emotional anchor, had increased experiences of this bias. They also concluded the illusion of transparency was exclusively present when experiencing obvious emotional states such as nervousness, disgust, and alarm (Gilovich et al., 1998).

Gilovich et al. can ultimately be credited for their influential, formative research and exploration of these egocentric biases highlighting the need to understand the spotlight effect and the illusion of transparency because they help us define ourselves in social settings $(1998,2000)$. In relation to social anxiety, further research that I will discuss has explored the negative effects of these biases on anxiety because of their heavy reliance on our unique phenomenological experiences as an anchor.

\section{SOCIAL ANXIETY}

Previously known as social phobia, social anxiety disorder (SAD) is not uncommon $(12.1 \%$ lifetime prevalence) to the general population where over 15 million American adults, or $6.8 \%$ of the U.S. population, are affected by it during any 12-month period (ADAA, 2020). The DSM-IV defines SAD as "a persistent fear of one or more social or performance situations in which the person is exposed to unfamiliar people or to possible scrutiny by others. The individual fears that he or she will act in a way (or show anxiety symptoms) that will be embarrassing and humiliating" (American Psychiatric Association, 2013, p. 202). Along with SAD inhibiting healthy functioning in social settings, it also makes those individuals more vulnerable to other clinical disorders like major depression and substance abuse (Ohayon \& Schatzberg, 2010; Randall et al., 2001). In addition, researchers have found the estimated number of feared social situations determined by an individual with SAD to be associated with comorbid major depression and suicidal ideation (Gabalawy, Cox, Clara, \& Mackenzie, 2010). Therefore, it is vital to explore possible cognitive vulnerabilities that sustain SAD because it can lead to other clinical disorders with similar or overlapping symptoms. 
Current cognitive-behavioral models of SAD argue that its development results from the use of self-focused information to predict what others think of them (Clark \& Wells, 1995), which is notable because both the spotlight effect and the illusion of transparency can then manifest from inaccurately assessed self-focused information. Research conducted by Brown and Stopa (2007) and Haikal and Hong (2010) suggests those with SAD use a heightened emotional anchor, based on a distorted internal appraisal caused by the spotlight effect and the illusion of transparency, which perpetuates the cyclical nature of anxiety.

\section{PRIOR RESEARCH}

\section{THE SPOTLIGHT EFFECT AS A MAINTAINING FACTOR}

A study conducted by Brown and Stopa (2007) aimed to explain how the spotlight effect and the illusion of transparency hinder the construction of a healthy sense of self by distorting internal and external appraisals in those with social anxiety. To do so, they recruited participants from a population of students at the University of Southampton who self-reported scores $\geq 36$ on the Brief Fear of Negative Evaluation Scale (BFNES: Leary, 1983). This cut-off point corresponded to the mean BFNES score and produced a sample of 60 participants who were moderately to highly socially anxious. The BFNES specifically measures fear of negative evaluation, which is a prominent component of social anxiety (American Psychiatric Association, 2013). In addition, self-reported presence and severity of depression over the previous two weeks were measured during the screening process using the Beck Depression Inventory-II (BDI-II: Beck et al., 1988), a valid instrument measuring depression symptom, producing a sample in the mild to moderate range.

A randomized between-subjects design was constructed, meaning each participant was randomly exposed to one condition in order to minimize learning effects, which would skew the results of the manipulation. Experimental conditions included either low or high social evaluation, where all participants performed a memory task and were told a group of experts would evaluate their task performance. Those in the high social-evaluative condition were openly videotaped, while the low social-evaluative condition was secretly recorded. After finishing the task, participants completed the BFNES and the BDI-II again, as well as the Self-Awareness and Task Performance Questionnaire (SATP-Q; Brown \& Stopa, 2007). The SATP-Q was administered to measure the spotlight effect and the illusion of transparency by including a modified version of the Situational Self-Awareness Scale (SSAS; Govern \& Marsch, 2001), which measures public and private self-awareness, as well as items regarding task performance. Finally, an independent assessor blind to the experimental conditions watched the recordings of the task and completed the assessor's version of the SATP-Q to compare participants' self-rating of public and private selfawareness with the assessors' rating of how well they could distinguish participants' selfawareness concerns during the task. To quantify public self-awareness, assessors rated how nervous participants appeared during the task and how poorly they performed in the task and quantified private self-awareness by rating how easily they could discern the participants' thoughts and feelings. 
To analyze the presence of the spotlight effect and the illusion of transparency, difference scores were calculated by subtracting participants' self-awareness scores on the SSAS items of the SATP-Q from the assessors scores on the same measures, such that higher positive differences would reflect higher levels of each bias. Using statistical analyses, the researchers found that participants in the high social-evaluative condition reported significantly higher levels of the spotlight effect than the low social-evaluative condition (Brown \& Stopa, 2007), consistent with their hypothesis. However, they found no significant difference in levels of the illusion of transparency between conditions (Brown \& Stopa, 2007). Further analyses found a significant difference between both biases for the high social-evaluative condition, such that the spotlight effect had a greater impact than the illusion of transparency, while participants in the low socialevaluative condition reported higher levels of the illusion of transparency compared to the spotlight effect, although this was not a significant difference (Brown \& Stopa, 2007). In sum, the spotlight effect was only significantly present in the high social-evaluative condition, while the illusion of transparency was not significantly influenced in either condition (Brown \& Stopa, 2007).

This study aimed to explore the extent to which socially anxious individuals overestimate how noticeable their internal and external state is to others. The findings expanded Gilovich et al.'s (1998, 2000) research, indicating and describing the presence of the spotlight effect and the illusion of transparency in non-socially anxious individuals, by concluding that these biases are present in socially anxious individuals to a higher degree (Brown \& Stopa, 2007). However, because of its consistency across social evaluative conditions, and contrary to Gilovich et al.'s (1998) idea that the illusion of transparency is a transitory state able to be manipulated, Brown and Stopa's (2007) findings suggested the illusion of transparency is more trait-like. That is, it would precede and predict anxiety rather than resulting from it, which with replication can have clinical implications in our understanding of social anxiety. Moreover, they propose the fear of public scrutiny, an indicator for SAD, increased focus on private self-awareness, which could explain the significant levels of the spotlight effect. Specifically, Clark and Wells (1995) found that socially anxious individuals form high standards for social performance, used as an inaccurate anchor, which leads to the belief that they cannot communicate a positive impression to others. Therefore, high socially evaluative contexts as highlighted in the current study leave socially anxious individuals more susceptible to high levels of the spotlight effect. The spotlight effect, more so than the illusion of transparency, acts as a vehicle for public and private self-awareness to stray from a healthy conception of the self as a social object such that it perpetuates the vicious cycle of social anxiety.

While Brown and Stopa's research (2007) provided speculative implications for the clinical understanding of social anxiety, the preliminary nature of their study included several limitations. First, their sample was limited to women, which makes it impossible to know if these findings are generalizable to men. There is a known meaningful difference between men and women, as women tend to be more prone to social anxiety (Caballo et al., 2014), so it could be argued this gender difference affects the presence of the spotlight effect and the illusion of transparency. Similarly, the sample was also limited to a student population, with a mean age of 19 years old, 
which does not allow for the examination of age-based effects. To combat these generalizability issues, the researchers proposed replication and recommended a more representative sample, possibly including several different social situations and clinical populations.

\section{THE ILLUSION OF TRANSPARENCY AS A MEDIATOR}

A study conducted by Haikal and Hong (2010) looked more specifically at the potential mediating effects of the spotlight effect and the illusion of transparency on the relationship between the fear of negative social evaluation (FNE) and looming cognitive style (LCS). To do so, they recruited undergraduate students from an existing pool who had previously participated in a related study at the National University of Singapore. During that study, self-reported data measuring FNE was collected using the Brief Fear of Negative Evaluation Scale (BFNE; Rodebaugh et al., 2004), a modified version of Leary's (1983) BFNES removing reverse-scored items to increase reliability and validity, and baseline LCS data was gathered with the Looming Maladaptive Style Questionnaire (LMSQ; Riskind et al., 2000). From this pool, the top 80 combined scores on the BFNE and the LMSQ were invited to participate in Haikal and Hong's (2010) study, creating an overall moderate- to high-risk sample of 52 participants.

The researchers utilized a 2 (social evaluation: low versus high) x 2 (temporal looming: low versus high) randomized between-subject design, which allowed experimental manipulation of both social evaluation and looming threat, permitting them to explore possible interaction effects caused by the spotlight effect and the illusion of transparency on anxiety symptoms. In other words, participants were randomly assigned to the high or low social evaluation condition and either the high or low temporal looming condition. Prior to the experiment, participants were readministered the BFNE and LMSQ to examine the stability of their scores, as well as measures to assess current mood and anxiety levels. The mean BFNE score in the sample was comparable to individuals diagnosed with SAD (Weeks et al., 2005), and the mean LMSQ score was lower than that of previous clinical samples (Riskind et al., 2007), but slightly higher than typically reported in normal samples. Anxiety levels were self-reported using the Beck Anxiety Inventory (BAI; Beck et al., 1988). Finally, after completing the task, participants in all conditions were asked to complete the same modified version of the SSAS utilized by Brown and Stopa (2007) to measure levels of the spotlight effect and the illusion of transparency.

All participants were tasked with performing a three- to five-minute-long speech introducing themselves which was video-recorded. However, the high social-evaluation condition was told their recordings would be evaluated by a group of communication experts and were shown how they would be recorded which was purposefully, but unknown to subjects, in an "up close," zoomed-in manner to evoke the belief that they would be closely evaluated (all cameras were later adjusted to be less zoomed-in after showing the participants so the recordings could be standardized). In contrast, the low social-evaluation condition was given the same speech task but were not told their recordings would be evaluated, and after the experiment, they were shown the standardized camera zoom instead of the close-up angle. 
Further, in the high temporal looming condition, the participants were told they had two minutes to prepare their speech and were given a digital stopwatch to increase the salience of temporal looming. After preparing they were asked to complete the BAI again. To standardize time between the conditions, this group was told there was an issue with the cameras and that they could further rehearse their speeches during the time researchers spent fixing it which allotted them a total of four minutes to prepare. The low temporal looming condition was told they would have some time to prepare but was not told specifically how long, and after giving these instructions, the researchers left the room for two minutes. Upon their return, they instructed participants to complete the BAI, after which they were told they would have an additional two minutes to prepare.

Statistical analyses of the data collected presented several interesting findings regarding the spotlight effect and the illusion of transparency. First, ANOVA analyses, which are commonly used to interpret and compare effects of more than one independent variable, found significant main effects of social evaluation and temporal looming on both the spotlight effect and the illusion of transparency (Haikal \& Hong, 2010). Specifically, participants in the high social evaluation condition showed higher levels of the spotlight effect and the illusion of transparency in comparison to the low social evaluation condition (Haikal \& Hong, 2010). The same was found in the temporal looming conditions (Haikal \& Hong, 2010). In other words, heightened fear of social evaluation and/or heightened temporal looming creates a context for both the spotlight effect and the illusion of transparency to become significantly present. Next, interactions between independent variables were investigated, and the researchers found an interaction between social evaluation and temporal looming significant in predicting residual change in BAI, which is calculated by finding the difference between baseline BAI and task BAI (Haikal \& Hong, 2010). This means when combined, high levels of social evaluation and high levels of temporal looming are predictive of increased levels of anxiety. The researchers also analyzed the relationship of residualized BAI to levels of the spotlight effect and the illusion of transparency and discovered a moderate positive correlation for both biases (Haikal \& Hong, 2010). This indicates that those experiencing increased levels of anxiety also experience increased levels of the spotlight effect and the illusion of transparency. However, because these relationships are present between the dependent variables of the study, regression analyses were utilized to discern if these biases had significant mediating effects, meaning they could explain the relationship between the experimental manipulations and residual change in BAI. Among the four cases where significant mediating effects could occur, only the illusion of transparency mediated the evaluation x looming interaction on the residual change in BAI (Haikal \& Hong, 2010). Therefore, the illusion of transparency explains how combined factors of socially demanding situations, FNE and LCS, predict heightened social anxiety.

Haikal and Hong (2010) ultimately expanded the findings of Brown and Stopa (2007), where it was found that higher levels of the spotlight effect are present in socially demanding contexts for those with social anxiety, by investigating the mediating effects of the spotlight effect and the illusion of transparency on the relationship between situational demands and anxiety levels. Their findings that higher levels of the spotlight effect are present in high social evaluative settings is 
consistent with the conclusions drawn from Brown and Stopa (2007) and highlights similar evidence that explains higher levels of the illusion of transparency being present under increased fear of negative social evaluation and temporal looming. However, little was found to support the hypothesis that the spotlight effect also had mediating effects, which the researchers argue could be because of the complex method used to calculate the difference in SSAS scores between participants and assessors, which could have masked more subtle effects. Further, in agreement with Gilovich et al. (1998), the findings of the present study support the idea that both biases stem from the anchoring-and-adjustment heuristic and argue they work to distort anchoring to a higher degree in socially demanding situations. Additionally, the interaction between social evaluation and temporal looming sustains Hirsch et al.'s (2006) combined cognitive bias hypothesis which proposes cognitive vulnerabilities may influence each other in the maintenance of a given disorder. While relatively new in social psychology, it is plausible that the spotlight effect and the illusion of transparency, based on Haikal and Hong (2010) and Brown and Stopa's (2007) research, play an important role in maintaining the social anxiety symptoms across both clinical and at-risk populations. Clinically, this implies socially anxious patients should be taught their perceptions are negatively distorted by these biases in order to decrease their influence on the cognitive vulnerabilities that underlie and maintain social anxiety.

While this study provided the necessary evidence to support and build off of the prior research mentioned, it presented several limitations. In comparison to Brown and Stopa's (2007) limitations, Haikal and Hong (2010) also faced the problem of generalizability due to their female undergraduate sample creating gender and age constraints. Secondly, the sample size was small (52 participants) due to a relatively weak return rate, creating the need for replication to validate their findings. Another issue with Haikal and Hong's (2010) sample comes from cultural differentiation in that the sample was composed of Asian identifying individuals; it is known that social anxiety is less common in Asian cultures than in traditional western cultures (Hofmann \& Hinton, 2014) which further illustrates the need for replication. Despite these limitations, their study added vital insight about the spotlight effect and the illusion of transparency in relation to social anxiety.

\section{FUTURE DIRECTIONS}

The findings described have laid the groundwork for understanding the impact the spotlight effect and the illusion of transparency have on socially anxious individuals, and several studies have since broadened the scope of this topic. For example, studies have looked at different clinical techniques to decrease the effects these biases have on social anxiety. Specifically, Macrae et al. (2016) found that altering mental imagery by having participants in their study imagine an upcoming social situation in third person reduced the spotlight effect and, as a result, anxiety levels. Additionally, both Brown and Stopa (2007) and Haikal and Hong (2010) suggested the video-feedback technique (Harvey et al., 2000) could be useful in cognitive-behavioral therapy (CBT) for social anxiety. Harvey et al. (2000) created it to provide corrective feedback for those experiencing social anxiety to alter their negative self-appraisals. Today video-feedback techniques have improved, and it is now a common tool for combating social anxiety symptoms 
(Shirotsuki, 2018). While mental imagery and video feedback have clinical implications known for decreasing anxiety, there is a lack of research on its usefulness in specifically decreasing the spotlight effect and the illusion of transparency. By exploring ways in which these biases can be decreased, the clinical implications can be generalizable to situations where they are present.

Moreover, the spotlight effect and the illusion of transparency in clinical disorders may be related to issues of comorbidity (Hirsch et al., 2006). That is, if these biases are found to help maintain different disorders, then decreasing bias effects could promote therapeutic effectiveness in treating comorbid disorders, which is when two or more disorders are present in an individual. For example, there is a prospective relationship between social anxiety and eating disorders (Levinson \& Rodebaugh, 2016), reliant partially on high FNE, increasing the spotlight effect and the illusion of transparency (Haikal \& Hong, 2010), that could be more effectively treated given further research regarding the combined cognitive bias hypothesis (Hirsch et al., 2006) and comorbidity.

Research conducted following both studies described in this paper highlight a) potential therapeutic techniques that decrease these cognitive vulnerabilities and b) the idea that these vulnerabilities could be implicated in comorbid disorders. Yet replication to support the validity of Brown and Stopa's (2006) and Haikal and Hong's (2010) is still necessary. It is highly recommended that replication occurs to better inform and support the current scope of the topic as above-mentioned.

In conclusion, the spotlight effect and the illusion of transparency are instrumental in the maintenance of social anxiety, proving the importance of broadening the related body of knowledge to gain a better understanding of how and to what extent they are damaging to a healthy sense of self in comorbid clinical populations. As social beings, perception is the greatest tool we have in navigating life, but distorted perceptions can become detrimental to one's wellbeing.

\section{ACKNOWLEDGMENTS}

The author would like to specifically thank Dr. David Condon for his feedback and continued support in the process of revising this piece.

\section{REFERENCES}

ADAA. (2020). Facts \& Statistics: Anxiety and Depression Association of America, ADAA. Facts \& Statistics | Anxiety and Depression Association of America, ADAA. https://adaa.org/understanding-anxiety/facts-statistics.

American Psychiatric Association. (2013). Diagnostic and statistical manual of mental disorders (5th ed.).

Beck, A. T., Epstein, N., Brown, G., \& Steer, R. A. (1988). An inventory for measuring clinical anxiety: Psychometric properties. Journal of Consulting and Clinical Psychology, 56(6), 
893-897. doi:10.1037/0022-006x.56.6.893

Brown, M. A., \& Stopa, L. (2007). The spotlight effect and the illusion of transparency in social anxiety. Journal of Anxiety Disorders, 21(6), 804-819. doi:10.1016/j.janxdis.2006.11.006

Caballo, V. E., Salazar, I. C., Irurtia, M. J., Arias, B., \& Hofmann, S. G. (2014). Differences in social anxiety between men and women across 18 countries. Personality and Individual Differences, 64, 35-40. doi:10.1016/j.paid.2014.02.013

Clark, D. M., \& Wells, A. (1995). A cognitive model of social phobia. In: R. G. Heimberg, M. R. Liebowitz, D. A. Hope, \& F. R. Schneier (Eds.), Social phobia: diagnosis, assessment, and treatment (pp. 69-93). New York: Guildford Press.

El-Gabalawy, R., Cox, B., Clara, I., \& Mackenzie, C. (2010). Assessing the validity of social anxiety disorder subtypes using a nationally representative sample. Journal of Anxiety Disorders, 24(2), 244-249. doi:10.1016/j.janxdis.2009.11.003

Gilovich, T., Savitsky, K., \& Medvec, V. H. (1998). The illusion of transparency: Biased assessments of others ability to read ones emotional states. Journal of Personality and Social Psychology, 75(2), 332-346. doi:10.1037/0022-3514.75.2.332

Gilovich, T., Medvec, V. H., \& Savitsky, K. (2000). The spotlight effect in social judgment: An egocentric bias in estimates of the salience of ones own actions and appearance. Journal of Personality and Social Psychology, 78(2), 211-222. doi:10.1037/0022-3514.78.2.211

Govern, J. M., \& Marsch, L. A. (2001). Situational Self-Awareness Scale. PsycTESTS Dataset. doi:10.1037/t14453-00o

Haikal, M., \& Hong, R. Y. (2010). The effects of social evaluation and looming threat on selfattentional biases and social anxiety. Journal of Anxiety Disorders, 24(3), 345-352. doi:10.1016/j.janxdis.2010.01.007

Harvey, A. G., Clark, D. M., Ehlers, A., \& Rapee, R. M. (2000). Social anxiety and selfimpression: cognitive preparation enhances the beneficial effects of video feed-back following a stressful social task. Behaviour Research and Therapy, 38, 1183-1192.

Hirsch, C. R., Clark, D. M., \& Mathews, A. (2006). Imagery and interpretations in social phobia: support for the combined cognitive biases hypothesis. Behavior therapy, 37(3), 223-236. https://doi.org/10.1016/j.beth.2006.02.001

Hofmann, S. G., \& Hinton, D. E. (2014). Cross-Cultural Aspects of Anxiety Disorders. Current Psychiatry Reports, 16(6). doi:10.1007/s11920-014-0450-3

Leary, M. R. (1983). Brief Fear of Negative Evaluation Scale. PsycTESTS Dataset. doi:10.1037/tooo48-00o

Levinson, C. A., \& Rodebaugh, T. L. (2016). Clarifying the prospective relationships between 
social anxiety and eating disorder symptoms and underlying vulnerabilities. Appetite, 107, 38-46. https://doi.org/10.1016/j.appet.2016.07.024

Macrae, C. Neil, et al. "Noticing Future Me: Reducing Egocentrism Through Mental Imagery.” Personality and Social Psychology Bulletin, vol. 42, no. 7, July 2016, pp. 855-863, doi:10.1177/0146167216644961.

Newell, A., Shaw, J. C., \& Simon, H. A. (1957). Empirical explorations of the logic theory machine. Empirical Explorations of the Logic Theory Machine: a Case Study in Heuristic. https://doi.org/10.1145/1455567.1455605

Ohayon, M. M., \& Schatzberg, A. F. (2010). Social phobia and depression: Prevalence and comorbidity. Journal of Psychosomatic Research, 68(3), 235-243. doi:10.1016/j.jpsychores.2009.07.018

Randall, C. L., Thomas, S., \& Thevos, A. K. (2001). Concurrent Alcoholism and Social Anxiety Disorder: A First Step Toward Developing Effective Treatments. Alcoholism: Clinical and Experimental Research, 25(2), 210-220. doi:10.1111/j.1530-0277.2001.tbo2201.x

Riskind, J. H., Williams, N. L., Gessner, T. L., Chrosniak, L. D., \& Cortina, J. M. (2000). The looming maladaptive style: Anxiety, danger, and schematic processing. Journal of Personality and Social Psychology, 79(5), 837-852. doi:10.1037/0022-3514.79.5.837

Riskind, J. H., Tzur, D., Williams, N. L., Mann, B., \& Shahar, G. (2007). Short-term predictive effects of the looming cognitive style on anxiety disorder symptoms under restrictive methodological conditions. Behaviour Research and Therapy, 45, 1765-1777.

Rodebaugh, T. L., Woods, C. M., Thissen, D. M., Heimberg, R. G., Chambless, D. L., \& Rapee, R. M. (2004). More Information From Fewer Questions: The Factor Structure and Item Properties of the Original and Brief Fear of Negative Evaluation Scale. Psychological Assessment, 16(2), 169-181. doi:10.1037/1040-3590.16.2.169

Rogers, C. R. (1974). A Theory of Therapy and Personality Change: As Developed in the Client Centered Framework. Perspectives in Abnormal Behavior, 341-351. https://doi.org/10.1016/b978-0-08-017738-0.50039-9

Ross, M., \& Sicoly, F. (1979). Egocentric biases in availability and attribution. Journal of Personality and Social Psychology, 37, 322-336.

Shirotsuki, K. (2018). Video Feedback Techniques Used in Social Anxiety Disorders. Cognitive Behavioral Therapy and Clinical Applications. https://doi.org/10.5772/intechopen.71278

Tversky, A., \& Kahneman, D. (1982). Judgment under uncertainty: Heuristics and biases. Judgment under Uncertainty, 3-20. https://doi.org/10.1017/cbo9780511809477.002

Weeks, J. W., Heimberg, R. G., \& Rodebaugh, T. L. (2008). The fear of positive evaluation scale: assessing a proposed cognitive component of social anxiety. Journal of Anxiety Disorders, 
22, 44-55. 